International Journal of Pure and Applied Mathematics

Volume 100 No. 2 2015, 263-278

ISSN: 1311-8080 (printed version); ISSN: 1314-3395 (on-line version)

url: http://www.ijpam.eu

doi: http://dx.doi.org/10.12732/ijpam.v100i2.8

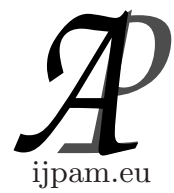

\title{
GROUP PURSUIT WITH PHASE CONSTRAINTS IN RECURRENT PONTRYAGIN'S EXAMPLE
}

\author{
N.N. Petrov ${ }^{1}$, N.A. Solov'eva ${ }^{2} \S$ \\ ${ }^{1,2}$ Udmurt State University \\ Universitetskaya st, 1, 426034, Izhevsk, RUSSIA
}

\begin{abstract}
We consider a generalized non-stationary Pontryagin's example under the same dynamic and inertial capabilities players and phase constraints on the state of the runaway. Boundary of phase constraints is not a "line of death" for the evader. Sufficient conditions for the capture of a group of pursuers one evader are obtained in this article.
\end{abstract}

AMS Subject Classification: 49N70, 49N75, 49N90

Key Words: differential game, group pursuit, the objective of capturing, Pontryagin's example, recurrent function

\section{Introduction}

Differential games of two players, first considered in the book of Isaacs [1], now present wide field of research [2]-[8]. Methods were developed for solving various classes of game problems: Isaaks' method, based on the analysis of a certain partial differential equation and its characteristics; Krasovskii's method of extremal guidans; Pontryagin's method and others. A natural generalization of differential games pursuit-evasion of two persons are games with a group of

Received: November 13, 2014

(C) 2015 Academic Publications, Ltd. url: www.acadpubl.eu

${ }^{\S}$ Correspondence author 
pursuers and one or several evaders [9]-[12]. These games are interesting from the theoretical point of view, they cannot be solved by theory for two-person games. One reason for this is, that the union sets of the reachability of all pursuers and the union of all target sets are sets, is non-convex and, furthermore, is not connected. On the other hand, there are some applications of these games to the problems of motion vehicles, collisions of avoidance for ships and others. In this case, the problem becomes much more complicated if the players on the state of the phase constraints are imposed. Among the large number of papers devoted to the problem of group pursuit with phase constraints, we mention the works [13]-[20]. In [13]-[17] there are the various tasks simple pursuit with phase constraints. The general problem of evasion with state constraints is considered in [18]. Sufficient mustache capture in stationary Pontryagin's example obtained in [19]. Terms of capture in an unsteady Pontryagin's example with phase constraints under other assumptions are presented in [20].

In this paper we consider the problem of persecution of a group of pursuers one evader at equal opportunities and inertial dynamic players. It is assumed that the evader in the game did not leave a convex polyhedral set, terminal sets - convex kompaty. Provided that some of the functions defined by the initial conditions and parameters of the game are recurrent, we obtain sufficient conditions for the solvability of persecution.

\section{Statement of the Problem}

In space $R^{k}(k \geq 2)$ we consider differential game $\Gamma(n, D) n+1$ objects: $n$ pursuers $P_{1}, \ldots, P_{n}$ and evader $E$.

The law of motion of each of the pursuers $P_{i}$ has the form

$$
x_{i}^{(l)}+a_{1}(t) x_{i}^{(l-1)}+a_{2}(t) x_{i}^{(l-2)}+\ldots+a_{l}(t) x_{i}=u_{i}, \quad u_{i} \in V
$$

The law of motion of evader $E$ has the form

$$
y^{(l)}+a_{1}(t) y^{(l-1)}+a_{2} y^{(l-2)}+\ldots+a_{l}(t) y=v, \quad v \in V,
$$

where $x_{i}, y_{j}, u_{i}, v_{j} \in R^{k}$, the functions $a_{1}(t), a_{2}(t), \ldots, a_{l}(t)$ are continuous $\left[t_{0}, \infty\right), V$ - convex compact. At $t=t_{0}$ the initial conditions are set

$$
x_{i}^{(q)}\left(t_{0}\right)=x_{i}^{q}, \quad y(q)\left(t_{0}\right)=y^{q},
$$

where $x_{i}^{0}-y^{0} \notin M_{i}$ for all $i, M_{i}$ is convex compact set. Here $i \in I=$ $\{1,2, \ldots, n\}, q=\{0,1, \ldots, l-1\}$. 
It is further assumed, that evader $E$ in the course of the game does not leave a convex set

$$
D=\left\{y: y \in R^{k},\left(p_{c}, y\right) \leq \mu_{c}, c=1,2, \ldots, r\right\},
$$

with non-empty interior, where $(a, b)$ is the scalar product of vectors $a$ and $b$, $p_{1}, \ldots, p_{r}$ are the unit vectors $R^{k}, \mu_{1}, \ldots, \mu_{r}$ are real numbers.

Instead (1)-(3), we consider to solve the equation

$$
z_{i}^{(l)}+a_{1}(t) z_{i}^{(l-1)}+a_{2}(t) z_{i}^{(l-2)}+\ldots+a_{l}(t) z_{i}=u_{i}-v,
$$

with initial conditions

$$
z_{i}^{(q)}\left(t_{0}\right)=z_{i}^{q}=x_{i}^{q}-y^{q} .
$$

Let $\varphi_{q}(t, s)\left(t \geq s \geq t_{0}\right)$ be the solution of the equation

$$
\omega^{(l)}+a_{1}(t) \omega^{(l-1)}+a_{2}(t) \omega^{(l-2)}+\ldots+a_{l}(t) \omega=0,
$$

with the initial conditions

$$
\begin{gathered}
\omega(s)=0, \ldots, \omega^{(q-1)}(s)=0, \omega^{(q)}(s)=1, \\
\omega^{(q+1)}(s)=0, \ldots, \omega^{(l-1)}(s)=0 .
\end{gathered}
$$

Suppose further

$$
\begin{aligned}
& \xi_{i}(t)=\varphi_{0}\left(t, t_{0}\right) z_{i}^{0}+\varphi_{1}\left(t, t_{0}\right) z_{i}^{1}+\ldots+\varphi_{l-1}\left(t, t_{0}\right) z_{i}^{l-1}, \\
& \eta(t)=\varphi_{0}\left(t, t_{0}\right) y^{0}+\varphi_{1}\left(t, t_{0}\right) y^{1}+\ldots+\varphi_{l-1}\left(t, t_{0}\right) y^{l-1} .
\end{aligned}
$$

Assume that $\xi_{i}(t) \notin M_{i}$ for all $i, t \geq t_{0}$, for if $\xi_{i}(\tau) \in M_{i}$ for some $i, \tau$, then the pursuer $P_{i}$ catches the evader $E$, supposing $u_{i}(t)=v(t)$.

Definition 1. We will say that a quasi-strategy $U_{i}$ of pursuer $P_{i}$, is given if a mapping $U_{i}\left(t, z^{0}, v_{t}(\cdot)\right)$ is defined that assigns to the initial state $z^{0}$, the point in time $t$, and an arbitrary past history of control $v_{t}(\cdot)$ of the evader $E$ such that $y(t) \in D$ for all $t \geq t_{0}$, a measurable function $u_{i}(t)$ with values in $V$.

Definition 2. There is a capture in the game $\Gamma(n, D)$ if there is a time $T\left(z^{0}\right)$ and quasi-strategies $U_{1}\left(t, z^{0}, v_{t}(\cdot)\right), \ldots, U_{n}\left(t, z^{0}, v_{t}(\cdot)\right)$ of pursuers $P_{1}, \ldots, P_{n}$ such that, for any measurable function $v(\cdot), v(t) \in V, y(t) \in D, t \in$ $\left[t_{0}, T\left(z^{0}\right)\right]$ there are numbers $s \in I$ and a time $\tau \in\left[t_{0}, T\left(z^{0}\right)\right]$, such that $z_{s}(\tau) \in M_{s}$. 
Definition 3. (see [21]) The function $f: R^{1} \rightarrow R^{k}$ is called recurrent if, for any $\varepsilon>0$, there exists $T(\varepsilon)>0$ such that for any $a, t \in R^{1}$, there exists $\tau(t) \in[a, a+T(\varepsilon)]$ for which it holds that

$$
\|f(t+\tau(t))-f(t)\|<\varepsilon
$$

Definition 4. The function $f:\left[t_{0}, \infty\right) \rightarrow R^{k}$ is called recurrent on $\left[t_{0}, \infty\right)$ if there exists a recurrent function $F: R^{1} \rightarrow R^{k}$ such that $f(t)=F(t)$ for all $t \in\left[t_{0}, \infty\right)$.

We define the functions

$$
\begin{aligned}
r(t, s) & =\left\{\begin{array}{l}
1, \text { if } \varphi_{l-1}(t, s) \geq 0, \\
-1, \text { if } \varphi_{l-1}(t, s)<0
\end{array} \quad\left(t_{0} \leq s \leq t\right),\right. \\
\lambda\left(v, \mu, b_{i}\right) & =\sup \left\{\lambda \mid-\lambda \mu\left(b_{i}-M_{i}\right) \cap(V-v) \neq \emptyset\right\}, \\
G_{i}\left(t, v(\cdot), b_{i}\right) & \left.=\int_{t_{0}}^{t}\left|\varphi_{l-1}(t, s)\right| \lambda\left(v(s), r(t, s), b_{i}\right)\right) d s, \\
F(t) & =\int_{t_{0}}^{t}\left|\varphi_{l-1}(t, s) d s\right| .
\end{aligned}
$$

\section{Capture Conditions}

Assumptions 1. 1) functions $\xi_{i}(t)$ are recurrent on $\left[t_{0}, \infty\right)$;

2) function $\eta(t)$ is bounded on $\left[t_{0}, \infty\right)$;

3) $\lim _{t \rightarrow \infty} F(t)=\infty$.

Assumptions 2. There are times $\tau_{i}^{0} \geq t_{0}$, are positive numbers $\varepsilon, \delta$ such that:

1) for all $i$ and for all $h_{i} \in S_{\varepsilon}\left(\xi_{i}\left(\tau_{i}^{0}\right)\right)$ is satisfied $h_{i} \notin M_{i}$;

2) for all $h_{i} \in S_{\varepsilon}\left(\xi_{i}\left(\tau_{i}^{0}\right)\right)$ inequality

$$
\min _{v} \max \left\{\max _{i} \lambda\left(v,+1, h_{i}\right), \max _{j}\left(p_{j}, v\right)\right\} \geq \delta,
$$




$$
\min _{v} \max \left\{\max _{i} \lambda\left(v,-1, h_{i}\right), \max _{j}\left(-p_{j}, v\right)\right\} \geq \delta
$$

where $S_{\varepsilon}(a)=\left\{x \in R^{k} \mid\|x-a\| \leq \varepsilon\right\}$.

Denote

$$
h=\left(h_{1}, h_{2}, \ldots, h_{n}\right), \quad S=S_{\varepsilon}\left(\xi_{1}\left(\tau_{1}^{0}\right)\right) \times S_{\varepsilon}\left(\xi_{2}\left(\tau_{2}^{0}\right)\right) \times \ldots \times S_{\varepsilon}\left(\xi_{n}\left(\tau_{n}^{0}\right)\right)
$$

Lemma 1. Suppose that assumptions 1, 2, $r=1$. Then there time $T \geq t_{0}$ such that for any admissible control $v(\cdot)$ evader $E$, any $h \in S$ there exists a number $m \in I$ for which $G_{m}\left(T, v(\cdot), h_{m}\right) \geq 1$.

Proof. Since control $v(t)$ of the evader $E$ is admissible, then for all $t \geq t_{0}$

$$
\left(p_{1}, y(t)\right) \leq \mu(t)=\mu_{1}-\left(p_{1}, \eta(t)\right)
$$

We define the set

$$
\begin{aligned}
& T^{+}(t)=\left\{\tau: \tau \in\left[t_{0}, t\right], \varphi_{l-1}(t, \tau) \geq 0\right\}, \\
& T^{-}(t)=\left\{\tau: \tau \in\left[t_{0}, t\right], \varphi_{l-1}(t, \tau)<0\right\}, \\
& T_{1}^{+}(t)=\left\{\tau: \tau \in T^{+}(t),\left(p_{1}, v(\tau)\right) \geq \delta\right\}, \\
& T_{2}^{+}(t)=\left\{\tau: \tau \in T^{+}(t),\left(p_{1}, v(\tau)\right)<\delta\right\}, \\
& T_{1}^{-}(t)=\left\{\tau: \tau \in T^{-}(t),\left(-p_{1}, v(\tau)\right) \geq \delta\right\}, \\
& T_{2}^{-}(t)=\left\{\tau: \tau \in T^{-}(t),\left(-p_{1}, v(\tau)\right)<\delta\right\} .
\end{aligned}
$$

Then

$$
\begin{aligned}
\int_{t_{0}}^{t} \varphi_{l-1}(t, s)\left(p_{1}, v(s)\right) d s= & \int_{T^{+}(t)} \varphi_{l-1}(t, s)\left(p_{1}, v(s)\right) d s \\
& +\int_{T^{-}(t)}\left(-\varphi_{l-1}(t, s)\right)\left(-p_{1}, v(s)\right) d s \\
= & \int_{T_{1}^{+}(t)} \varphi_{l-1}(t, s)\left(p_{1}, v(s)\right) d s+\int_{T_{2}^{+}(t)} \varphi_{l-1}(t, s)\left(p_{1}, v(s)\right) d s \\
& +\int_{T_{1}^{-}(t)}\left(-\varphi_{l-1}(t, s)\right)\left(-p_{1}, v(s)\right) d s
\end{aligned}
$$




$$
\begin{aligned}
& +\int_{T_{2}^{-}(t)}\left(-\varphi_{l-1}(t, s)\right)\left(-p_{1}, v(s)\right) d s \\
\geq & \delta \int_{T_{1}^{+}(t)} \varphi_{l-1}(t, s) d s-\int_{T_{2}^{+}(t)} \varphi_{l-1}(t, s) d s \\
& +\delta \int_{T_{1}^{-}(t)}\left(-\varphi_{l-1}(t, s)\right) d s-\int_{T_{2}^{-}(t)}\left(-\varphi_{l-1}(t, s)\right) d s \\
= & \delta \int_{T_{1}^{+}(t) \cup T_{1}^{-}(t)}\left|\varphi_{l-1}(t, s)\right| d s-\int_{T_{2}^{+}(t) \cup T_{2}^{-}(t)}\left|\varphi_{l-1}(t, s)\right| d s .
\end{aligned}
$$

Deduce

$$
\begin{gathered}
\delta \int_{T_{1}^{+}(t) \cup T_{1}^{-}(t)}\left|\varphi_{l-1}(t, s)\right| d s-\int_{T_{2}^{+}(t) \cup T_{2}^{-}(t)}\left|\varphi_{l-1}(t, s)\right| d s \leq \mu(t), \\
\quad \int_{T_{1}^{+}(t) \cup T_{1}^{-}(t)}\left|\varphi_{l-1}(t, s)\right| d s+\int_{T_{2}^{+}(t) \cup T_{2}^{-}(t)}\left|\varphi_{l-1}(t, s)\right| d s=F(t) .
\end{gathered}
$$

The last two relations imply that

$$
\int_{T_{2}^{+}(t) \cup T_{2}^{-}(t)}\left|\varphi_{l-1}(t, s)\right| d s \geq \frac{\delta F(t)-\mu(t)}{1+\delta} .
$$

Further, we have

$$
\begin{aligned}
\max _{i \in I} G_{i}\left(t, v(\cdot), h_{i}\right) & =\max _{i \in I} \int_{t_{0}}^{t}\left|\varphi_{l-1}(t, s)\right| \lambda\left(v(s), r(t, s), h_{i}\right) d s \\
& \geq \frac{1}{n} \int_{t_{0}}^{t}\left|\varphi_{l-1}(t, s)\right| \sum_{i \in I} \lambda\left(v(s), r(t, s), h_{i}\right) d s \\
& \geq \frac{\delta}{n} \int_{T_{2}^{+}(t) \cup T_{2}^{-}(t)}\left|\varphi_{l-1}(t, s)\right| d s \\
& \geq \frac{\delta}{n}\left(\frac{\delta F(t)-\mu(t)}{1+\delta}\right) .
\end{aligned}
$$

Since $F(t) \rightarrow \infty$ at $t \rightarrow \infty$ and $\mu(t)$ is bounded, then we obtain the desired result. The lemma is proved. 
Define the number $T_{0}$

$$
T_{0}=\min \left\{t \geq t_{0}: \inf _{v(\cdot)} \min _{h \in S} \max _{i \in I} G_{i}\left(t, v(\cdot), h_{i}\right) \geq 1\right\} .
$$

Assumptions 3. There are times $\tau_{i} \geq T_{0}$ such that:

1) $\xi_{i}\left(\tau_{i}\right) \in S_{\varepsilon}\left(\xi_{i}\left(\tau_{i}^{0}\right)\right)$ for all $i$;

2) $\inf _{v(\cdot)} \max _{i} G_{i}\left(\tau_{i}, v(\cdot), \xi_{i}\left(\tau_{i}\right)\right) \geq 1$.

Remarks. a) the existence of $\tau_{i}$ in paragraph 1 of assumptions 3 guaranteed assumption that recurrence functions $\xi_{i}(t)$;

b) if the assumption 3 of all $\tau_{i}=\tau$, paragraph 2 of this assumption is made automatically by Lemma 1 .

Theorem 1. Suppose that assumptions 1, 2, 3, $r=1$. Then in the game $\Gamma(n, D)$ capture occurs.

Proof. By the Cauchy's formula, the solution of problem (4), (5) for all $t \geq t_{0}$ for any admissible control has the form

$$
z_{i}(t)=\xi_{i}(t)+\int_{t_{0}}^{t} \varphi_{l-1}(t, s)\left(u_{i}(s)-v(s)\right) d s .
$$

Let $\tau_{i}$ - points satisfying assumption $3, v(s), s \in\left[t_{0}, T_{1}\right]$ be an arbitrary admissible control of the evader $E$, where $T_{1}=\max _{i} \tau_{i}$. Consider the function

$$
H(t)=1-\max _{i} \int_{t_{0}}^{t}\left|\varphi_{l-1}\left(\tau_{i}, s\right)\right| \lambda\left(v(s), r\left(\tau_{i}, s\right), \xi_{i}\left(\tau_{i}\right)\right) d s .
$$

We denote by $\tau_{0} \geq t_{0}$ is the first root of this function. Note that, by the assumption 2, the time $\tau_{0}$ exists, wherein $\tau_{0} \leq \tau_{i}$ for at least one of $i$. Furthermore, there is an index $m$ such that

$$
1-\int_{t_{0}}^{\tau_{0}}\left|\varphi_{l-1}\left(\tau_{m}, s\right)\right| \lambda\left(v(s), r\left(\tau_{m}, s\right), \xi_{m}\left(\tau_{m}\right)\right) d s=0 .
$$


For $j \neq m$ is also denoted $t_{j}$ - times for which the condition (7), if such times exist. By Filippov's lemma [22] for each $i$ there are measurable functions $m_{i}(s)$, $u_{i}(s), s \in\left[t_{0}, T_{1}\right]$, which for each fixed $s$, the solution of the equation

$$
\lambda\left(v(s), r\left(\tau_{j}, s\right), \xi_{i}\left(\tau_{j}\right)\right)\left(\xi_{i}\left(\tau_{i}\right)-m_{i}\right)=u_{i}-v(s) .
$$

We specify the control of pursuers $P_{i}$, in the following way:

$$
\begin{aligned}
& u_{i}(t)=v(t)-\lambda\left(v(t), r\left(\tau_{i}, t\right), \xi_{i}\left(\tau_{i}\right)\right)\left(\xi_{i}\left(\tau_{i}\right)-m_{i}(t)\right), \quad t \in\left[t_{0}, \min \left\{t_{i}, T_{1}\right\}\right], \\
& u_{i}(t)=v(t), t \in\left(\min \left\{t_{i}, T_{1}\right\}, T_{1}\right] .
\end{aligned}
$$

Then

$$
\begin{aligned}
z_{i}\left(\tau_{i}\right)= & \xi_{i}\left(\tau_{i}\right)+\int_{t_{0}}^{\tau_{i}} \varphi\left(\tau_{i}, s\right)\left(u_{i}(s)-v(s)\right) d s \\
= & \xi_{i}\left(\tau_{i}\right)-\int_{t_{0}}^{\tau_{i}}\left|\varphi_{l-1}\left(\tau_{i}, s\right)\right| \lambda\left(v(s), r\left(\tau_{i}, s\right), \xi_{i}\left(\tau_{i}\right)\right)\left(\xi_{i}\left(\tau_{i}\right)-m_{i}(s)\right) d s \\
= & \xi_{i}\left(\tau_{i}\right)\left(1-\int_{t_{0}}^{t_{i}}\left|\varphi_{l-1}\left(\tau_{i}, s\right)\right| \lambda\left(v(s), r\left(\tau_{i}, s\right), \xi_{i}\left(\tau_{i}\right)\right) d s \mid\right. \\
& +\int_{t_{0}}^{t_{i}}\left|\varphi_{l-1}\left(\tau_{i}, s\right)\right| \lambda\left(v(s), r\left(\tau_{i}, s\right), \xi_{i}\left(\tau_{i}\right)\right) m_{i}(s) d s .
\end{aligned}
$$

(7) it follows that

$$
z_{m}\left(\tau_{m}\right)=\int_{t_{0}}^{t_{m}}\left|\varphi_{l-1}\left(\tau_{m}, s\right)\right| \lambda\left(v(s), r\left(\tau_{m}, s\right), \xi_{m}\left(\tau_{m}\right)\right) m_{m}(s) d s \in M_{m}
$$

The theorem is proved.

\section{Capture Conditions in the Case of $V=S_{1}(0)$}

We denote $\operatorname{Int} X, \operatorname{co} X$ respectively the interior and the convex hull of the set $\mathrm{X}$,

$$
\lambda\left(M_{i}, v\right)=\sup \left\{\lambda \geq 0 \lambda M_{i} \cap(V-v) \neq \emptyset\right\} .
$$


Lemma 2. Let $V=S_{1}(0), Q_{i}, i \in I$ - convex compact sets $R^{k}, 0 \notin Q_{i}$ for all $i$. Then

$$
\delta^{+}=\min _{v} \max \left\{\max _{i}, \lambda\left(Q_{i}, v\right), \max _{j}\left(p_{j}, v\right)\right\}>0
$$

if and only if

$$
0 \in \operatorname{Intco}\left\{Q_{1}, \ldots, Q_{n}, p_{1}, \ldots, p_{r}\right\} .
$$

Proof. Note that (see [9], p. 46)

$$
\lambda\left(Q_{i}, v\right)=\max _{q_{i} \in Q_{i}} \frac{\left(q_{i}, v\right)+\sqrt{\left(q_{i}, v\right)^{2}+\left\|q_{i}\right\|^{2}\left(1-\|v\|^{2}\right)}}{\left\|q_{i}\right\|^{2}} .
$$

Suppose that $\delta^{+}=0$. Then there exists an $v_{0},\left\|v_{0}\right\|=1$ such that

$$
\lambda\left(Q_{i}, v_{0}\right)=0 \text { for all } i,\left(p_{j}, v_{0}\right) \leq 0 \text { for all } j .
$$

Therefore, the following inequalities

$$
\left(q_{i}, v_{0}\right) \leq 0 \text { for all } i, q_{i} \in Q_{i},\left(p_{j}, v_{0}\right) \leq 0 \text { for all } j .
$$

Therefore, 0 and $\operatorname{co}\left\{Q_{1}, \ldots, Q_{n}, p_{1}, \ldots, p_{r}\right\}$ separable. Hence $0 \notin \operatorname{Intco}\left\{Q_{1}, \ldots, Q_{n}, p_{1}, \ldots, p_{r}\right\}$.

We now assume that $0 \notin \operatorname{Intco}\left\{Q_{1}, \ldots, Q_{n}, p_{1}, \ldots, p_{r}\right\}$. Then 0 and

$$
\operatorname{co}\left\{Q_{1}, \ldots, Q_{n}, p_{1}, \ldots, p_{r}\right\}
$$

separable. Consequently, there exists $v_{0},\left\|v_{0}\right\|=1$ such that the inequalities (8). Hence $\lambda\left(Q_{i}, v_{0}\right)=0$ for all $i$. Therefore $\delta^{+}=0$. The lemma is proved.

Corollary 1. Let $V=S_{1}(0), Q_{i}, i \in I$ - convex compact sets $R^{k}, 0 \notin Q_{i}$ for all $i$. Then

$$
\delta^{-}=\min _{v} \max \left\{\max _{i}, \lambda\left(-Q_{i}, v\right), \max _{j}\left(-p_{j}, v\right)\right\}>0
$$

if and only if

$$
0 \in \operatorname{Intco}\left\{Q_{1}, \ldots, Q_{n}, p_{1}, \ldots, p_{r}\right\} .
$$

Assumptions 4. There $\tau_{i}^{0} \geq t_{0}$ such that

$$
0 \in \operatorname{Intco}\left\{\xi_{i}\left(\tau_{i}^{0}\right)-M_{i}, i \in I, p_{1}, \ldots, p_{r}\right\}
$$


Lemma 3. Suppose that assumptions 1, 4. Then there are positive numbers $\varepsilon, T(\varepsilon)$ for which the following propositions:

1) for all $h_{i} \in S_{\varepsilon}\left(\xi_{i}\left(\tau_{i}^{0}\right)\right)$ the following inclusion

$$
h_{i} \notin M_{i}, 0 \in \operatorname{Intco}\left\{h_{i}-M_{i}, i \in I, p_{1}, \ldots, p_{r}\right\} ;
$$

2) for each $t \geq t_{0}$ there are moments $\tau_{i} \in[t, t+T(\varepsilon)]$, that $\xi_{i}\left(\tau_{i}\right) \in S_{\varepsilon}\left(\xi_{i}\left(\tau_{i}^{0}\right)\right)$.

The first proposition follows from the properties of the strict separation of convex sets, and the second assertion follows from the properties of recurrent functions.

We fix $\varepsilon>0$ and $T(\varepsilon)>0$, so that there have been propositions of Lemma 4.

Lemma 4. Let $V=S_{1}(0)$ and assumption 4 is fulfiled. Then, for any $h \in S$ we have the inequalities

$$
\begin{aligned}
\delta^{+}(h) & =\min _{v} \max \left\{\max _{i} \lambda\left(h_{i}, 1, v\right), \max _{j}\left(p_{j}, v\right)\right\}>0, \\
\delta^{-}(h) & =\min _{v} \max \left\{\max _{i} \lambda\left(h_{i},-1, v\right), \max _{j}\left(-p_{j}, v\right)\right\}>0, \\
\delta & =\min _{h \in S} \min \left\{\delta^{+}(h), \delta^{-}(h)\right\}>0 .
\end{aligned}
$$

Proof. Let $h \in S$. Then are fulfiled for the sets $Q_{i}=h_{i}-M_{i}$ the conditions of Lemmas 3 and 4. Therefore, $\delta^{+}(h)>0$. By Lemma 1.3.13 [9, p.30] function $\delta^{+}(h)$ is continuous on $S$. Therefore, by the Weierstrass's theorem $\min _{h \in S} \delta^{+}(h)>$ 0. Similarly $\min _{h \in S} \delta^{-}(h)>0$. Consequently, $\delta>0$. The lemma is proved.

Definition 5. (see [23]) The vectors $a_{1}, \ldots, a_{s}$ form a positive basis of $R^{k}$, if, for any $x \in R^{k}$, there exist positive real numbers $\gamma_{1}, \ldots, \gamma_{s}$ such that

$$
x=\gamma_{1} a_{1}+\cdots+\gamma_{s} a_{s} .
$$

Theorem 2. (see [23]) Vectors $a_{1}, \ldots, a_{s}$ a positive basis to form $R^{k}$ if and only if

$$
0 \in \operatorname{Intco}\left\{a_{1}, \ldots, a_{s}\right\}
$$

Lemma 5. Let $Q_{i}, i \in I$ be convex compact sets $R^{k}, 0 \notin Q_{i}$ for all $i$ and the following conditions: 
1) $0 \in \operatorname{Intco}\left\{Q_{1}, \ldots, Q_{n}, p_{1}, \ldots p_{r}\right\}$;

2) the number of elements $\bigcup_{i=1}^{n} Q_{i}$ at least to $k$;

3) in the set $\bigcup_{i=1}^{n} Q_{i}$ exist $k$ linearly independent vectors.

Then there exist $p \in R^{k}, \mu \in R^{1}$ such that:

1) $D \subset D_{1}=\left\{z \mid z \in R^{k},(p, z) \leq \mu\right\}$;

2) $0 \in \operatorname{Intco}\left\{Q_{1}, \ldots, Q_{n}, p\right\}$.

Proof. According to the conditions of Lemma there are $q_{1}, \ldots, q_{s} \in \bigcup_{i=1}^{n} Q_{i}$ such that

$$
0 \in \operatorname{Intco}\left\{q_{1}, \ldots, q_{s}, p_{1}, \ldots, p_{r}\right\} .
$$

We can assume that $s \geq k$ and the vectors $q_{1}, \ldots, q_{k}$ linearly independent. By theorem 2 , the vectors $q_{1}, \ldots, q_{s}, p_{1}, \ldots, p_{r}$ form a positive basis. Therefore, there exist positive integers $\alpha_{1}, \ldots, \alpha_{s}, \beta_{1}, \ldots, \beta_{r}$ such that

$$
0=\alpha_{1} q_{1}+\cdots+\alpha_{s} q_{s}+\beta_{1} p_{1}+\cdots+\beta_{r} p_{r} .
$$

Consider the vector $p=\beta_{1} p_{1}+\cdots+\beta_{r} p_{r}$. We show that the set of $q_{1}, \ldots, q_{s}, p$ forms a positive basis.

Let $x \in R^{k}$. Since $q_{1}, \ldots q_{k}$ is a basis of $R^{k}$, then there exist numbers $\gamma_{1}, \ldots, \gamma_{k}$ such that

$$
x=\gamma_{1} q_{1}+\cdots+\gamma_{k} q_{k} .
$$

By (9) we obtain that for any $d \in R^{1}$ equality

$$
x=\gamma_{1} q_{1}+\cdots+\gamma_{k} q_{k}+d\left(\alpha_{1} q_{1}+\cdots+\alpha_{s} q_{s}+\beta_{1} p_{1}+\cdots+\beta_{r} p_{r}\right) .
$$

Taking $d>0$ such that the inequality $\gamma_{c}+d \alpha_{c}>0$ for all $c=1, \ldots, k$ obtain that

$$
x=\gamma_{1}^{0} q_{1}+\gamma_{s}^{0} q_{s}+d p
$$

and wherein all the coefficients are positive. Consequently, $q_{1}, \ldots, q_{s}, p$ constitute a positive basis. So $0 \in \operatorname{Intco}\left\{q_{1}, \ldots, q_{s}, p\right\}$ and therefore

$$
0 \in \operatorname{Intco}\left\{Q_{1}, \ldots, Q_{s}, p\right\} .
$$

Consider the set

$$
D_{1}=\left\{x \mid x \in R^{k},(p, x) \leq \mu\right\},
$$


where $\mu=\beta_{1} \mu_{1}+\cdots+\beta_{r} \mu_{r}$. Then $D \subset D_{1}$. Note that if $p=0$, then $D_{1}=R^{k}$. The lemma is proved.

Assumptions 5. For any $h \in S$ in the set $\bigcup_{i=1}^{n}\left(h_{i}-M_{i}\right)$ there is a $k$ linear independent vectors.

Corollary 2. Suppose that assumptions 1, 4, 5. Then, for any $h \in S$ exist a vector $p(h) \in R^{k}$ and the number of $\mu(h) \in R^{1}$ such that:

1) $0 \in \operatorname{Intco}\left\{h_{i}-M_{i}, i \in I, p(h)\right\}$;

2) $D \subset D_{1}=\left\{z \mid z \in R^{k},(p(h), z) \leq \mu(h)\right\}$.

Lemma 6. Let $V=S_{1}(0)$ and assumptions 1, 4, 5 be fulfiled. Then there is the time $T \geq t_{0}$ such that for any admissible control $v(\cdot)$ of the evader $E$ in the game $\Gamma\left(n, D_{1}\right)$, any $h \in S$ there exists a number $m \in I$ such that

$$
G_{m}\left(T, v(\cdot), h_{m}\right) \geq 1
$$

where $D_{1}$ is defined in corollary fact 2 .

Proof. Suppose $h \in S$. By Lemma 5, we have $\delta>0$. Therefore, the assumption that the conditions 2. Therefore, we can apply Lemma 1, which implies the assertion. The lemma is proved.

Theorem 3. Let the assumptions of $1,4,5$, and there are $\tau_{i} \geq T_{0}$ such that:

1) $\xi_{i}\left(\tau_{i}\right) \in S_{\varepsilon}\left(\xi_{i}\left(\tau_{i}^{0}\right)\right)$;

2) $\inf _{v(\cdot)} \max _{i} G_{i}\left(\tau_{i}, v(\cdot), \xi_{i}\left(\tau_{i}\right)\right) \geq 1$ in the game $\Gamma\left(n, D_{1}\right)$.

Then in the game $\Gamma\left(n, D_{1}\right)$ capture occurs.

The validity of this assertion follows from theorem 1 .

Corollary 3. Suppose that all the conditions of theorem 3. Then in the game $\Gamma(n, D)$ capture occurs. 


\section{Examples}

Example 1. Suppose that $k=2, n=1, M_{1}=\{0\}, p_{1}=(1,0), \mu_{1}=2$, $x^{0}=(0,0), y^{0}=(1,0), t_{0}=0$, the system (3),(4) has the form

$$
\dot{z}=u-v, u, v \in V=\left\{\left(v_{1}, v_{2}\right) \mid v_{1}, v_{2} \in[-1,1]\right\} .
$$

Proposition 1. In the game $\Gamma(1, D)$ capture occurs.

Proof. In this case fulfiled $\varphi_{0}(t)=1, \xi(t)=z^{0}=(-1,0), F(t)=t$ and therefore assumption 1 . Furthermore assumption 2 , as $\delta=0,5$. Assumption 3 is automatically satisfied. Theorem 1 is applicable.

Example 2. Suppose that the system (3),(4) has the form

$$
\dot{z}_{i}=u_{i}-v, z_{i}(0)=z_{i}^{0}
$$

For this example, assumption 1.

Proposition 2. Let $V=S_{1}(0)$, in the set $\left(\bigcup_{i=1}^{n}\left(z_{i}^{0}-M_{i}\right)\right.$ there is a $k$ linearly independent vectors and

$$
0 \in \operatorname{Intco}\left\{z_{i}^{0}-M_{i}, i \in I, p_{1}, \ldots, p_{r}\right\} .
$$

In the game $\Gamma(n, D)$ capture occurs.

Proposition 3. (see [13]) Let $V=S_{1}(0), D$ be polyhedron, $M_{i}=\{0\}$ for all $i$ and $n \geq k$.

Then in the game $\Gamma(n, D)$ capture occurs.

Example 3. Let $V=S_{1}(0)$, in the system (3),(4) $l=1, t_{0}=0$, the function $a_{1}(t)$ has the form

$$
a_{1}(t)=\left\{\begin{array}{l}
0, \text { if } t \in[0,2 \pi] \\
\sin t, \text { if } t>2 \pi
\end{array}\right.
$$

Then the function $\varphi_{0}(t)$ has the form

$$
\varphi_{0}(t)=\left\{\begin{array}{l}
1, \text { if } t \in[0,2 \pi], \\
e^{1-\cos t}, \text { if } t>2 \pi .
\end{array}\right.
$$


Function $\varphi_{0}(t)$ is recurrent, but is not almost periodic (see [21]). Assumption 1 is satisfied.

Proposition 4. Let $D=R^{k}$, the initial position $z_{i}^{0}$ such that

$$
0 \in \operatorname{Intco}\left\{z_{i}^{0}-M_{i}, i \in I\right\} .
$$

Then in the game $\Gamma(n, D)$ capture occurs.

Example 4. Let $V=S_{1}(0)$, the system (3),(4) has the form

$$
\ddot{z}_{i}+\frac{2}{3 t} \dot{z}_{i}+\frac{1}{9 t^{2 / 3}} z_{i}=u_{i}-v
$$

and $t_{0}=8 \pi^{2}$. Then

$$
\begin{aligned}
\varphi_{0}(t, s) & =\cos (\sqrt[3]{t}-\sqrt[3]{s}) \\
\varphi_{1}(t, s) & =3 s^{2 / 3} \sin (\sqrt[3]{t}-\sqrt[3]{s}) \\
\xi_{i}(t) & =z_{i}^{0} \cos (\sqrt[3]{t})+12 \pi^{2} z_{i}^{1} \sin (\sqrt[3]{t}) .
\end{aligned}
$$

Recurrence functions $\xi_{i}(t)$ from the results of (see [21]).

Proposition 5. Assume that there exists time $\tau \in\left[t_{0}, \infty\right)$ such that the set $\bigcup_{i=1}^{n}\left(\xi_{i}(\tau)-M_{i}\right)$ there is a $k$ linearly independent vectors and

$$
0 \in \operatorname{Intco}\left\{\xi_{i}(\tau)-M_{i}, i \in I, p_{1}, \ldots, p_{r}\right\} .
$$

Then in the game $\Gamma(n, D)$ capture occurs.

Taking as $\tau_{i}^{0}=t_{0}=8 \pi^{2}$, fairly

Proposition 6. Let the initial position $z_{i}^{0}$ such that the set $\bigcup_{i=1}^{n}\left(z_{i}^{0}-M_{i}\right)$ there is a $k$ linearly independent vectors and

$$
0 \in \operatorname{Intco}\left\{z_{i}^{0}-M_{i}, i \in I, p_{1}, \ldots, p_{r}\right\} .
$$

Then in the game $\Gamma(n, D)$ capture occurs.

Note that for this example are not fulfilled the conditions of [20]. 


\section{Acknowledgments}

This work was supported by RFBR (project 15-01-02602) and the Ministry of Education and Science of the Russian Federation within the base portion.

\section{References}

[1] R. Isaacs, Differential Games, John Wiley and Sons, New York (1965).

[2] L.S. Pontryagin, Selected scientific works (in Russian), Vol. 2, Nauka, Moscow, (1988).

[3] A. Blaquiere, F. Gerard, G. Leitmann, Quantitative and Qualitative Differential Games, Academic Press, New York (1969).

[4] N. N. Krasovskii, Game Problems of Contact of Motions (in Russian), Nauka, Moscow (1970).

[5] A. Friedman, Differential Games, John Wiley and Sons, New York, (1971).

[6] N. N. Krasovskii, A. I. Subbotin, Positional Differential Games (in Russian), Nauka, Moscow (1974).

[7] O. Hajek, Pursuit Games, Academic Press, New York (1975).

[8] G. Leitmann, Cooperative and Noncooperative Many-Player Differential Games, Springer-Verlag, Vienna, Austria (1974).

[9] L. A. Petrosyan, Differential Games of Pursuit (in Russian), Leningrad University Press, Leningrad (1977).

[10] A. I. Subbotin, A. G. Chentsov, Optimization of a Guarantee in Problems of Control (in Russian), Nauka, Moscow (1981).

[11] A.A. Chikrii, Conflict controlled prosesses,Kluwer Acad. Publ.,Boston, Lomdon, Dordrecht (1997).

[12] N. L. Grigorenko, Mathematical methods of control a few dynamic processes (in Russian), Moscow State University, Moscow (1990).

[13] A.I. Blagodatskikh, N.N. Petrov, Conflict interaction of groups of controlled objects (in Russian), Udmurt State University, Izhevsk (2009). 
[14] N. Yu. Satimov, B.B. Rikhsiev, Methods of solving the problem of avoiding encounter in mathematical control theory (in Russian), Fan, Tashkent (2000).

[15] R. P. Ivanov, Simple pursuit in a compact set (in Russian), Doklady Akademii Nauk SSSR, 254, No.6 (1978), 1318-1321.

[16] N.N. Petrov, On the nonstationary problem of group pursuit with phase constraints, Automation and Remote Control, 75, No.8 (2014), 1525-1531, doi: 10.1134/S0005117914080153.

[17] N. N. Petrov, K.A. Shchelchkov, About the problem of group persecution in linear differential games with a simple matrix and state constraints, International Journal of Pure and Applied Mathematics, 92, No. 1 (2014), 13-26, doi: 10.12732/ijpam.v92i1.2.

[18] F. L. Chernous'ko, The one problem of evasion from many pursuers (in Russian), Prikl. Mat. Mekh., 40, No.1 (1976), 14-24.

[19] W. Rzymovski, On the game of $n+1$ cars, J. Math. Analys Appl., 99, (1984), 109-122.

[20] F.L. Chernous'ko, V.L. Zak, On Differential Games of Evasion from Many Pursuers, J. Optimiz. Theory Appl., 46, No.4 (1985), 461-470.

[21] N. N. Petrov, Multiple capture in Pontryagin's example with phase constraints, Journal of Applied Mathematics and Mechanics, 61, No.5 (1997), 725-732, doi: 10.1016/S0021-8928 (97)00095-6.

[22] A. S. Bannikov, N.N. Petrov, On a nonstationary problem of group pursuits Proceedings of the Steklov Institute of Mathematics, 271, No. 1 (Suppl) (2010), 41-52, doi: 10.1134/S0081543810070047.

[23] V.I. Zubov, The theory of recurrent functions, Siberian Mathematical Journal, 3, No. 4 (1962), 532-560.

[24] A. F. Filippov, On some questions of the theory of optimal control, Vestnik Moskovskogo Universiteta. Seriya 1. Matematika. Mekhanika, No. 6 (1959), 25-32.

[25] N. N. Petrov, On the controllability of autonomous systems, Differential Equations, 4, No. 4 (1968), 606-617. 